\title{
CONTRIBUTIONS TO THE FLORA OF CIMARRON COUNTY AND THE BLACK MESA AREA
}

\author{
Amy K. Buthod \\ Oklahoma Biological Survey \\ University of Oklahoma \\ Norman, OK 73019 \\ amybuthod@ou.edu
}

\author{
Bruce W. Hoagland \\ Oklahoma Biological Survey \\ Department of Geography and \\ Environmental Sustainablity \\ University of Oklahoma \\ Norman, OK 73019
}

Keywords: flora, Cimarron County, Black Mesa, vascular plants, rare plants

\begin{abstract}
This paper reports the results of recent collection activities in Cimarron County, including the Black Mesa area, in the state of Oklahoma. A total of 331 taxa in 60 families were collected. Two-hundred and six genera, 279 species and 52 infraspecific taxa were identified. The largest families were the Poaceae with 72 taxa and the Asteraceae with 63. Thirty-six exotic taxa were collected (10.9\% of the flora), including two species new to Oklahoma: Scorzonera laciniata and Ranunculus testiculatus. Forty-six taxa tracked by the Oklahoma Natural Heritage Inventory were found.

\section{INTRODUCTION}

Cimarron County has long been recognized as a botanically significant region in Oklahoma. A total of 95 vascular plants tracked by the Oklahoma Natural Heritage Inventory (ONHI) occur in the county (Oklahoma Natural Heritage Inventory 2013). Included among these is Asclepias uncialis Greene, which, prior to 1996, was listed as a likely candidate for federal listing as threatened or endangered by the U.S. Fish and Wildlife Service (United States Department of the Interior 1993). Before this survey, nineteen of the tracked taxa had an $\mathrm{ONHI}$ ranking of $\mathrm{SH}$, meaning that reports of occurrences are older than twenty years (Oklahoma Natural Heritage Inventory 2013; NatureServe 2015). The number of taxa in Cimarron County that are rare at the state level is due in part to the presence of Black Mesa, an extension of the Mesa de Maya, which extends for $72 \mathrm{~km}$ from east of Raton, New Mexico, though Colorado and into northwestern Cimarron

County. The eastern-most extension of the Rocky Mountain foothill vegetation is present in the area; Rogers (1953) found it to be "an excellent example of the intergradation of the flora of the great plains with that of the Rocky Mountain foothills". Our intent for this work was to relocate the rare taxa, update their ONHI ranks, and, hopefully, expand our knowledge of the area's current flora.

The earliest botanical collections from the Black Mesa region were made in 1820 by Edwin James, botanist for Major Stephen Long's expedition to the Rocky Mountains. Eighty-four years later, Per Axel Rydberg, author of Flora of Colorado (1906) and Flora of the Rocky Mountains and Adjacent Plains (1917), botanized in the area. The first thorough botanical inventory of the Mesa de Maya was completed by Rogers (1953). From 1947 and 1949, he collected along the mesa in Colorado, New Mexico and Oklahoma, as well as from some of the secondary mesas in the area (Rogers 1953). According
\end{abstract}


to a list published in 1953, Rogers collected 267 taxa from 51 families in Oklahoma, but in a later work (1954) he notes that "approximately five-hundred were found, or could be found". U. T. Waterfall collected at Black Mesa and in Cimarron County within the same time period, adding approximately 30 taxa to the state's flora (Waterfall 1949, 1950a, b). James K. McPherson completed an inventory with the sole focus of Black Mesa in the early 1990s, reporting 236 taxa from 58 families $(2003 \mathrm{a}, \mathrm{b})$. His collecting activities were confined to the areas of the mesa on the property belonging to the state of Oklahoma (Township 6N, Range 1E, Sections 28-33 and Township 5N, Range 1E, Section 6). Patricia Folley (2003) supplemented the McPherson list with collections made from 1994 through 2003. Folley collected over a wider area than McPherson, surveying the state park around Lake Etling, the roadsides leading to the park and mesa, and some private lands, including Tesequite Canyon (Folley 2003). She found an additional 49 taxa from 25 families. Other botanists have contributed to the knowledge of the Black Mesa/Cimarron County flora over the years, including Delzie Demaree, who worked in the area in the 1930s, George Goodman (from the late 1930s through the early 1970s), John and Connie Taylor (1960s and 1970s), and Larry Magrath in the 1980s (Oklahoma Vascular Plants Database 2015).

\section{STUDY SITE}

Cimarron County falls within the High Plains and the Cimarron River Valley geomorphic provinces (Curtis et al. 2008). The High Plains province consists of flat uplands over Tertiary-era Dakota sandstones and is found throughout most of the county (Rogers 1953). The Cimarron River Valley is found in the northeastern part of the county and is distinguished by dissected valleys of Mesozoic-era shale and sandstone. The Black Mesa, the flat, eroded remnant of a Tertiary-era lava flow, is located in this area (Curtis et al. 2008). The highest point in Oklahoma, at $1515 \mathrm{~m}$, is on the mesa. Rolling, low hills and canyons surround the mesa.

Four soil associations occur within Cimarron County. Travessilla-Kim soils are only found in the northeastern corner of the county. They consist of "loam, calcareous, and humus-poor soils on steep slopes" (Carter and Gregory 2008). Dalhart-Vona soils are found primarily in the southern half of the county; these are "very deep loamy soils on gentle slopes" (Carter and Gregory 2008). Sherm-Ulysses type soils dominate the eastern half of the county. These soils are "very deep, silty and clayey, humus-rich soils on gentle slopes" (Carter and Gregory 2008). Conlen-Pastura-Plack soils are the least common soil type in the county; they consist of "loamy and calcareous soils on moderately steep slopes" (Carter and Gregory 2008). Potential vegetation types in Cimarron County include shortgrass high plains, sandsage grassland, piñon pine/juniper mesa, and bottomland forest (Duck and Fletcher 1943; Hoagland 2008).

Cimarron County has a dry climate, falling within Trewartha's steppe or semiarid type (1968). Average annual precipitation ranges from $38-50 \mathrm{~cm}$, with most falling from May through August. Thunderstorms occur in the spring and summer. Average temperature is $13-14^{\circ} \mathrm{C}$. The average high (in July) is $34^{\circ} \mathrm{C}$, and the average low (in January) is $-7^{\circ} \mathrm{C}$. Southsouthwesterly winds are dominant and relative humidity ranges from $29-84 \%$. Over $70 \%$ of days are sunny (Oklahoma Climatological Survey 2015).

\section{METHODS}

Plants were collected at 100 sites throughout Cimarron County (Fig. 1; Table 1). Collection sites were chosen based on location information from the Oklahoma Natural Heritage Inventory Database and 


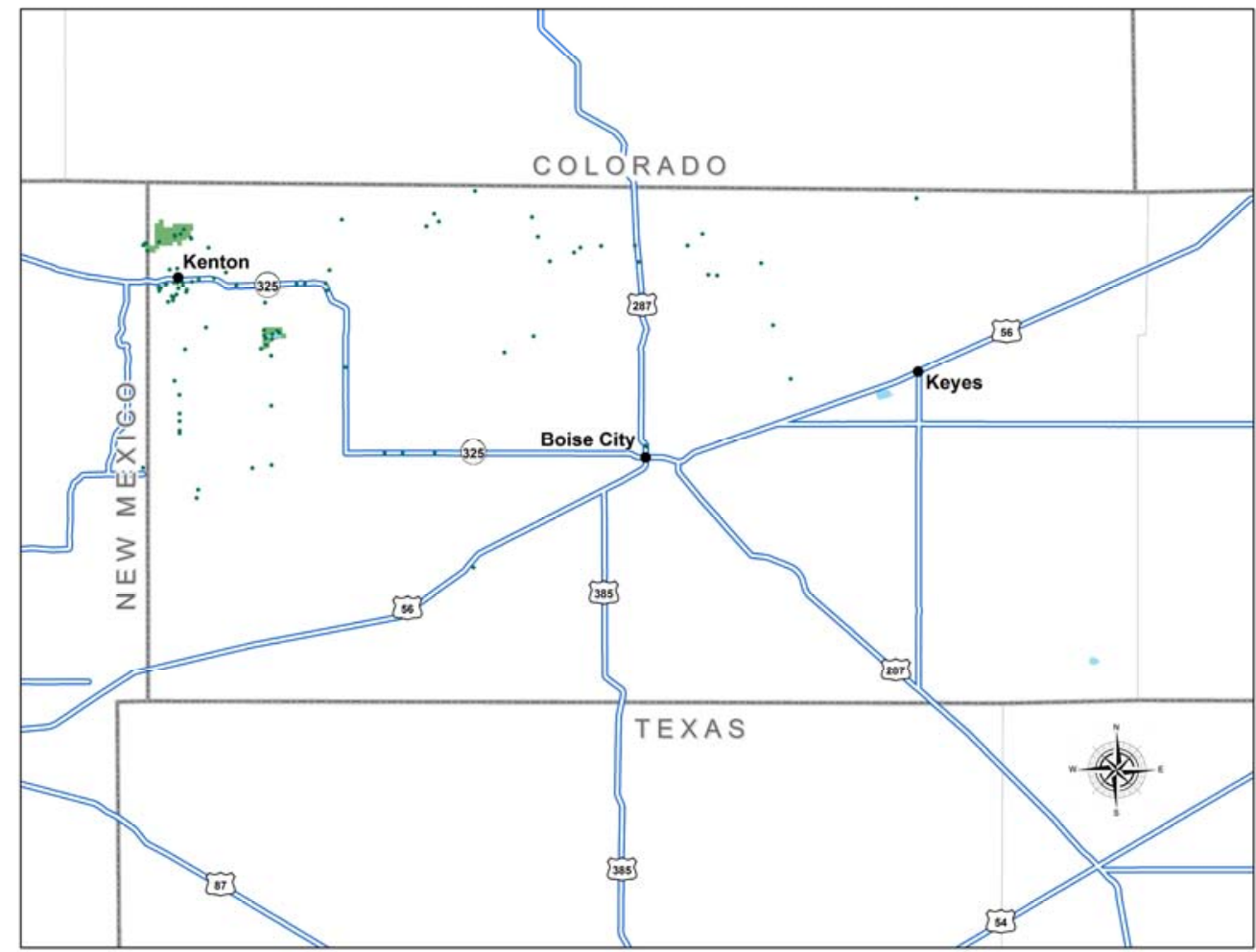

Figure 1 Cimarron County, Oklahoma. Dots indicate collection sites. Shaded areas indicate Black Mesa Nature Preserve lands. Map by Todd Fagin, Oklahoma Biological Survey.

the Oklahoma Vascular Plants Database. Additional collections were also made opportunistically. Coordinates of each site were collected using a Garmin GPSmap 76Cx unit. Sites were located between latitudes N36.98989 and N36.62313 and longitudes W102.67913 and W102.68063. Elevations ranged from $1118 \mathrm{~m}$ to $1513 \mathrm{~m}$. Field work began in March of 2013, with subsequent monthly trips until September. An additional trip was made in May of 2014. One example of each taxon encountered was collected and processed according to standard herbarium protocols. Specimens were deposited at the Robert Bebb Herbarium (OKL) at the University of Oklahoma. Manuals used to identify plants included Great Plains Flora Association (1986), Tyrl et al. (2010) and Allred and Ivey (2012); the collections of the Robert Bebb Herbarium were also used to verify identifications. Taxonomy follows the Integrated Taxonomic Information System (2015). Duration and nativity to Oklahoma were determined using the PLANTS Database (USDA-NRSC 2015); if the information from PLANTS was ambiguous, Taylor and Taylor (1991) was consulted. Vegetation classifications were assigned based on Hoagland (2000).

\section{RESULTS AND DISCUSSION}

Three-hundred and thirty-one taxa in 60 families were collected in this study (Appendix A). Two-hundred and six genera, 279 species, and 52 infraspecific taxa were identified. Two-hundred thirty-one taxa were perennials; there were 96 annuals and four biennials. Thirty-six taxa were nonnative to Oklahoma, including two species new to the state (Scoryonera laciniata in the Asteraceae and Ranunculus testiculatus in the 
Ranunculaceae); non-native taxa accounted for $10.9 \%$ of the total flora. The Poaceae had the greatest number of exotic taxa with 11; the Brassicaceae had five. The largest families were the Poaceae with 72 taxa and the Asteraceae with 63. Forty-six taxa tracked by the Oklahoma Natural Heritage inventory were found (Table 2). Asclepias uncialis, the former candidate for federal listing, was not located.

Vegetation classes encountered in this study included the Artemisia filifolia/Sporobolus cryptandrus-Schizachyrium scoparium shrubland association. It is found on sandy soils and stabilized dunes in the northwestern and central portions of the study site. Associated taxa included Andropogon gerardii ssp. hallii, Abronia fragrans, and Eriogonum annuum (Duck and Fletcher 1943; Hoagland 2000).

Two intergrading variations of shortgrass prairie were noted. The Bouteloua curtipendula-B. gracilis-B. dactyloides herbaceous association is found on rocky slopes and well-drained soils in the southern part of the study area (Duck and Fletcher 1943; Hoagland 2000). Plants found here included Mublenbergia torreyi, Ratibida columnifera, and Sphaeraclea coccinea. The Boutelona gracilisHilaria jamesii herbaceous association is found in northwestern Cimarron County on slopes and uplands (Hoagland 2000). Plants found in this type included Cylindropuntia imbricata, Melampodium leucanthum, and Zinnia grandiflora.

The Boutelona gracilis-Hilaria jamesii herbaceous association intergrades with the fourth vegetation type, the Juniperus monosperma woodland alliance. This alliance includes the Juniperus monosperma/Bouteloua curtipendula woodland association and the Juniperus monosperma-Pinus edulis/Bouteloua curtipendula woodland association and is found in northwestern Cimarron County. Plants from this type included Boutelona gracilis, Cercocarpus montanus, and Prunus virginiana (Hoagland 2000).

Herbaceous wetland vegetation was found at only a few sites, including those with seeps, lakes, and intermittently flowing streams and rivers. Plants found in this vegetation type included Polypogon monspeliensis, Populus deltoides, Salix exigua, and Tamarix chinensis. Vegetation of disturbed areas includes taxa found around lawns, stock tanks, campgrounds, parking lots, and gravel pits. Plants in this vegetation type included Conyza canadensis, Descurainia sophia, Kochia scoparia, and Malva neglecta.

Table 1 Collection sites in Cimarron County

$\begin{array}{lll}\text { Latitude } & \text { Longitude } & \begin{array}{c}\text { Township, Range, and } \\ \text { Section }\end{array} \\ 36.623130 & -102.68063 & \text { Sec. } 24-T 2 N-R 3 E \\ 36.690420 & -102.95001 & \text { Sec. 33-T3N-R1E } \\ 36.698390 & -102.9484 & \text { Sec. } 28-T 3 N-R 1 E \\ 36.719380 & -102.89576 & \text { Sec. } 13-T 3 N-R 1 E \\ 36.719660 & -103.00208 & \text { Sec. } 18-T 3 N-R 1 E \\ 36.722180 & -102.877 & \text { Sec. } 18-T 3 N-R 2 E\end{array}$

Amy K. Buthod and Bruce W. Hoagland 


\begin{tabular}{|c|c|c|}
\hline 36.733790 & -102.7183 & Sec. $15-T 3 N-R 3 E$ \\
\hline 36.733800 & -102.76698 & Sec. 17-T3N-R3E \\
\hline 36.733920 & -102.74949 & Sec. 16-T3N-R3E \\
\hline 36.739900 & -102.51231 & Sec. $10-T 3 N-R 5 E$ \\
\hline 36.741100 & -102.51344 & Sec. $10-T 3 N-R 5 E$ \\
\hline 36.753940 & -102.96656 & Sec. 4-T3N-R1E \\
\hline 36.756350 & -102.96655 & Sec. 4-T3N-R1E \\
\hline 36.765380 & -102.96653 & Sec. 32-T4N-R1E \\
\hline 36.772640 & -102.96652 & Sec. 32-T4N-R1E \\
\hline 36.780300 & -102.87736 & Sec. 30-T4N-R2E \\
\hline 36.790710 & -102.96668 & Sec. 29-T4N-R1E \\
\hline 36.804340 & -102.97145 & Sec. $20-\mathrm{T} 4 \mathrm{~N}-\mathrm{R} 1 \mathrm{E}$ \\
\hline 36.806220 & -102.37202 & Sec. $13-T 4 N-R 6 E$ \\
\hline 36.817480 & -102.80509 & Sec. 13-T4N-R2E \\
\hline 36.829480 & -102.87738 & Sec. 7-T4N-R2E \\
\hline 36.832480 & -102.65052 & Sec. 8-T4N-R4E \\
\hline 36.835430 & -102.96116 & Sec. 9-T4N-R1E \\
\hline 36.836080 & -102.88737 & Sec. 6-T4N-R2E \\
\hline 36.840080 & -102.88219 & Sec. 6-T4N-R2E \\
\hline 36.845780 & -102.87656 & Sec. 5-T4N-R2E \\
\hline 36.846420 & -102.88263 & Sec. 6-T4N-R2E \\
\hline 36.848380 & -102.62216 & Sec. 3-T4N-R4E \\
\hline 36.849240 & -102.88435 & Sec. 6-T4N-R2E \\
\hline 36.850360 & -102.87642 & Sec. 31-T5N-R2E \\
\hline 36.850360 & -102.87642 & Sec. 31-T5N-R2E \\
\hline
\end{tabular}




\begin{tabular}{|c|c|c|}
\hline 36.851790 & -102.86967 & Sec. 32-T5N-R2E \\
\hline 36.853670 & -102.87138 & Sec. 32-T5N-R2E \\
\hline 36.854090 & -102.88454 & Sec. 31-T5N-R2E \\
\hline 36.856980 & -102.94078 & Sec. 34-T5N-R1E \\
\hline 36.859200 & -102.38917 & Sec. 34-T5N-R6E \\
\hline 36.881280 & -102.88344 & Sec. 19-T5N-R2E \\
\hline 36.882050 & -102.97772 & Sec. $20-T 5 N-R 1 E$ \\
\hline 36.883330 & -102.97295 & Sec. $20-T 5 N-R 1 E$ \\
\hline 36.886460 & -102.97238 & Sec. $20-T 5 N-R 1 E$ \\
\hline 36.887550 & -102.97424 & Sec. $20-T 5 N-R 1 E$ \\
\hline 36.889260 & -102.96963 & Sec. $20-T 5 N-R 1 E$ \\
\hline 36.891690 & -102.96015 & Sec. $21-T 5 N-R 1 E$ \\
\hline 36.892660 & -102.98643 & Sec. $19-T 5 N-R 1 E$ \\
\hline 36.893120 & -102.82283 & Sec. $22-\mathrm{T} 5 \mathrm{~N}-\mathrm{R} 2 \mathrm{E}$ \\
\hline 36.893790 & -102.95947 & Sec. $16-T 5 N-R 1 E$ \\
\hline 36.895370 & -102.9677 & Sec. $17-T 5 N-R 1 E$ \\
\hline 36.895760 & -102.98476 & Sec. $18-T 5 N-R 1 E$ \\
\hline 36.895960 & -102.98691 & Sec. $18-T 5 N-R 1 E$ \\
\hline 36.897520 & -102.91134 & Sec. $13-T 5 N-R 1 E$ \\
\hline 36.897950 & -102.96324 & Sec. $16-T 5 N-R 1 E$ \\
\hline 36.898540 & -102.98034 & Sec. $17-T 5 N-R 1 E$ \\
\hline 36.899190 & -102.97931 & Sec. $17-T 5 N-R 1 E$ \\
\hline 36.899370 & -102.8527 & Sec. $16-T 5 N-R 2 E$ \\
\hline 36.899560 & -102.84465 & Sec. $16-T 5 N-R 2 E$ \\
\hline 36.899830 & -102.82454 & Sec. $15-T 5 N-R 2 E$ \\
\hline
\end{tabular}

Amy K. Buthod and Bruce W. Hoagland 


\begin{tabular}{|c|c|c|}
\hline 36.900190 & -102.96891 & Sec. $17-T 5 N-R 1 E$ \\
\hline 36.900640 & -102.97209 & Sec. $17-T 5 N-R 1 E$ \\
\hline 36.901170 & -102.96404 & Sec. $16-T 5 N-R 1 E$ \\
\hline 36.901410 & -102.95449 & Sec. $16-T 5 N-R 1 E$ \\
\hline 36.903700 & -102.94789 & Sec. $16-T 5 N-R 1 E$ \\
\hline 36.904800 & -102.93303 & Sec. $15-T 5 N-R 1 E$ \\
\hline 36.907530 & -102.44369 & Sec. 7-T5N-R6E \\
\hline 36.908160 & -102.45214 & Sec. 7-T5N-R6E \\
\hline 36.910440 & -102.92143 & Sec. $11-T 5 N-R 1 E$ \\
\hline 36.912730 & -102.82081 & Sec. $11-T 5 N-R 2 E$ \\
\hline 36.913450 & -102.97624 & Sec. 8-T5N-R1E \\
\hline 36.914270 & -102.96875 & Sec. 8-T5N-R1E \\
\hline 36.919640 & -102.4009 & Sec. $10-T 5 N-R 6 E$ \\
\hline 36.920710 & -102.51988 & Sec. 9-T5N-R5E \\
\hline 36.921370 & -102.60638 & Sec. $10-T 5 N-R 4 E$ \\
\hline 36.921370 & -102.60638 & Sec. $10-T 5 N-R 4 E$ \\
\hline 36.929980 & -102.58279 & Sec. 1-T5N-R4E \\
\hline 36.931820 & -102.99784 & Sec. 6-T5N-R1E \\
\hline 36.934710 & -102.9383 & Sec. 3-T5N-R1E \\
\hline 36.934710 & -102.93839 & Sec. 3-T5N-R1E \\
\hline 36.934850 & -102.57666 & Sec. 1-T5N-R4E \\
\hline 36.936330 & -102.55646 & Sec. 21-T6N-R5E \\
\hline 36.936870 & -102.52358 & Sec. 33-T6N-R5E \\
\hline 36.936880 & -102.47233 & Sec. 36-T6N-R5E \\
\hline 36.937150 & -103.0018 & Sec. 6-T5N-R1E \\
\hline
\end{tabular}




\begin{tabular}{|c|c|c|}
\hline 36.938120 & -103.00098 & Sec. 31-T6N-R1E \\
\hline 36.938800 & -103.00023 & Sec. 31-T6N-R1E \\
\hline 36.939080 & -102.99954 & Sec. 31-T6N-R1E \\
\hline 36.940380 & -102.98649 & Sec. 31-T6N-R1E \\
\hline 36.943380 & -102.95534 & Sec. 33-T6N-R1E \\
\hline 36.944330 & -102.95544 & Sec. 33-T6N-R1E \\
\hline 36.945420 & -102.618 & Sec. 34-T6N-R4E \\
\hline 36.945760 & -102.97118 & Sec.32-T6N-R1E \\
\hline 36.947060 & -102.97128 & Sec. 32-T6N-R1E \\
\hline 36.947930 & -102.96566 & Sec. 33-T6N-R1E \\
\hline 36.948080 & -102.45784 & Sec. 31-T6N-R6E \\
\hline 36.952680 & -102.96242 & Sec. 28-T6N-R1E \\
\hline 36.955610 & -102.72656 & Sec. 27-T6N-R3E \\
\hline 36.960120 & -102.71428 & Sec. 27-T6N-R3E \\
\hline 36.962150 & -102.80867 & Sec. 26-T6N-R2E \\
\hline 36.964600 & -102.62363 & Sec. $28-T 6 N-R 4 E$ \\
\hline 36.967830 & -102.71885 & Sec. 22-T6N-R3E \\
\hline 36.982940 & -102.24962 & Sec. 13-T6N-R7E \\
\hline 36.989890 & -102.67913 & Sec. 13-T6N-R3E \\
\hline
\end{tabular}


Table 2 Taxa located during this study that are tracked by the Oklahoma Natural Heritage Inventory (Oklahoma Natural Heritage Inventory 2013; NatureServe Explorer 2015). Status ranks are on a $1-5$ scale, with a 1 indicating the taxa is critically imperiled. G ranks are at the global level and $\mathrm{S}$ ranks are at the subnational or state level. Infraspecific taxa are assigned a $\mathrm{T}$ rank. A taxon with NR indicates that it has not been ranked at the global level (NatureServe 2015). Highlighted taxa were re-ranked as a result of this survey.

\begin{tabular}{|c|c|c|}
\hline Family & Taxon & Ranking \\
\hline Amaranthaceae & Krascheninnikovia lanata (Pursh) A. Meeuse \& A. Smit & S1G5 \\
\hline Apocynaceae & Asclepias macrotis Torr. & S1G4 \\
\hline Asteraceae & Ambrosia confertiflora DC. & S1G5 \\
\hline Asteraceae & Artemisia carruthii Alph. Wood ex Carruth. & S2G4? \\
\hline Asteraceae & Brickellia brachyphylla (A. Gray) A. Gray & S1G5 \\
\hline Asteraceae & Brickellia californica (Torr. \& A. Gray) A. Gray & S1G5 \\
\hline Asteraceae & Brickellia eupatorioides (L.) Shinners var. & S1G5T5 \\
\hline Asteraceae & Ericameria nauseosa (Pall. ex Pursh) G.L. Nesom \& & S1G5T5 \\
\hline Asteraceae & Picradeniopsis woodhousei (A. Gray) Rydb. & S2G4G5 \\
\hline Asteraceae & Solidago velutina DC. ssp. sparsiflora (A. Gray) Semple & S1G5?TNR \\
\hline Boraginaceae & Cryptantha cinerea (Greene) Cronquist var. & S2G5T5? \\
\hline Boraginaceae & Cryptantha thyrsiflora (Greene) Payson & S2G4 \\
\hline Cactaceae & Cylindropuntia imbricata (Haw.) F.M. Knuth & S2G5 \\
\hline Cactaceae & Echinocereus reichenbachii (Terscheck ex Walp.) J.N. & S3G5 \\
\hline Cactaceae & Echinocereus viridiflorus Engelm. & S1G5 \\
\hline Cactaceae & Escobaria vivipara (Nutt.) Buxb. & S1G5 \\
\hline Cactaceae & Opuntia polyacantha Haw. var. polyacantha & S2G5T5 \\
\hline Convolvulaceae & Cuscuta umbellata Kunth & S1G5 \\
\hline Crossomataceae & Glossopetalon spinescens A. Gray var. & S1G5TNR \\
\hline Cupressaceae & Juniperus monosperma (Engelm.) Sarg. & S2G4G5 \\
\hline Fabaceae & Dalea formosa Torr. & S2G5 \\
\hline
\end{tabular}




\begin{tabular}{|c|c|c|}
\hline Fabaceae & Dalea jamesii (Torr.) Torr. \& A. Gray & S1G5 \\
\hline Fabaceae & Desmanthus cooleyi (Eaton) Trel. & S2G5 \\
\hline Fabaceae & Hoffmannseggia drepanocarpa A. Gray & S2G5 \\
\hline Fabaceae & Lupinus plattensis $\mathrm{S}$. Watson & S1G4 \\
\hline Grossulariaceae & Ribes cereum Douglas & S1G5 \\
\hline Malvaceae & Sphaeralcea angustifolia (Cav.) G. Don & S2G5 \\
\hline Nyctaginaceae & Abronia fragrans Nutt. ex Hook. & S2G5 \\
\hline Papaveraceae & Argemone squarrosa Greene & S1G4 \\
\hline Pinaceae & Pinus edulis Engelm. & S1G5 \\
\hline Plantaginaceae & Penstemon fendleri Torr. \& A. Gray & S1G5T4? \\
\hline Poaceae & Aristida arizonica $\mathrm{V}$ asey & S1G4 \\
\hline Poaceae & Bouteloua barbata Lag. & S1G5 \\
\hline Poaceae & Bouteloua eriopoda (Torr.) Torr. & S1G5 \\
\hline Poaceae & Hesperostipa neomexicana (Thurb.) Barkworth & S1G4G5 \\
\hline Poaceae & Hilaria jamesii (Torr.) Benth. & S1G5 \\
\hline Poaceae & Mublenbergia phleoides (Kunth) Columbus & S1G5 \\
\hline Poaceae & Mublenbergia porteri Scribn. ex Beal & S1G5 \\
\hline Poaceae & Mublenbergia torreyi (Kunth) Hitchc. ex Bush & S1G4 \\
\hline Poaceae & Piptatherum micranthum (Trin. \& Rupr.) Barkworth & S1G5 \\
\hline Polygonaceae & Eriogonum jamesii Benth. & S1G5 \\
\hline Polygonaceae & Eriogonum lachnogynum Torr. ex Benth. & S1G4? \\
\hline Polygonaceae & Eriogonum tenellum Torr. & S1G5 \\
\hline Rosaceae & Cercocarpus montanus Raf. & S1G5 \\
\hline Rosaceae & Rubus deliciosus Torr. & S1G4? \\
\hline Selaginellaceae & Selaginella underwoodii Hieron. & S1G5? \\
\hline
\end{tabular}

Amy K. Buthod and Bruce W. Hoagland 


\section{DISCUSSION}

One-hundred sixty taxa from 46 families reported in the Rogers, McPherson, and Folley studies were not found (Appendix B), and only 46 of the 95 taxa tracked by the Oklahoma Natural Heritage Inventory were located. One explanation for this difference is land access. For instance, we were not able to collect in Tesequite Canyon, which is known to have populations of tracked taxa (Oklahoma Natural Heritage Inventory 2015), as was done in the Folley study. We were uncomfortable botanizing along some of the public roads, as well. Another explanation could be that vegetation changes have occurred in the area. Vegetation analysis by Graham et al. (unpubl. data) indicates a decrease in the amount of grassland/herbaceous vegetation and an increase in forest/shrubland since 1992. This is most probably due to the increased amount of cholla (Cylindropuntia imbricata) in the area.

The most likely explanation for our results, however, is drought. Cimarron County is considered to be the epicenter of the exceptional drought experienced by the High Plains regions of northern Texas, southwestern Kansas, northeastern New Mexico, southeastern Colorado, and the northwestern Oklahoma panhandle (Lindsey 2008; South Central Climate Science Center 2013). Throughout the survey period, western Cimarron County experienced exceptional, extreme, or extreme/severe drought (National Oceanic and Atmospheric Administration et al. 2015). Rogers (1953) stated that the "severe drouth of the 1930s had a disturbing effect on the vegetation", but noted a "great recovery" in the following decade. Although the National Weather Service predicts that the drought status for the area will likely be removed, another "great recovery" is unlikely (U. S. Geological Survey 2014). The area could be as much as $5^{\circ} \mathrm{C}$ hotter by the end of the century, and decreases in precipitation, runoff, and amounts of soil water storage are also likely (U. S.

Geological Survey 2014).

\section{ACKNOWLEDGEMENTS}

This work was supported by a grant from the Department of Interior, U. S. Fish and Wildlife Service. The authors wish to thank Todd Fagin (Oklahoma Biological Survey/Department of Geography and Environmental Sustainability, University of Oklahoma) for assistance with map preparation.

\section{LITERATURE CITED}

Allred, K.W. and R.D. Ivey. 2012. Flora Neomexicana III: An Illustrated Identification Manual. Self-published.

Carter, B.J. and M.S. Gregory. 2008. Soil map of Oklahoma. In: Earth Sciences and Mineral Resources of Oklahoma. Johnson, K.S. and K.V. Luza (eds.). Norman $(\mathrm{OK})$ : Oklahoma Geological Survey.

Curtis, N.M., W.E. Ham, and K.S. Johnson. 2008. Geomorphic provinces of Oklahoma. In: Earth Sciences and Mineral Resources of Oklahoma. Johnson, K.S. and K.V. Luza (eds.) Norman (OK): Oklahoma Geological Survey.

Duck, L.G. and J.B. Fletcher. 1943. A Survey of the Game and Fur-Bearing Animals of Oklahoma. Oklahoma City (OK): Oklahoma Department of Wildlife Conservation.

Folley, P.A. 2003. Additions to Black Mesa flora study. Oklahoma Native Plant Record 3:19-22.

Great Plains Flora Association. 1986. Flora of the Great Plains. Lawrence (KS): University of Kansas Press.

Hess, W.J. 2002. Nolina. pp. 415-422. In: Flora of North America North of Mexico. Vol. 26. Flora of North American Editorial Committee (eds.). New York and Oxford. 
Hoagland, B.W. 2000. The vegetation of Oklahoma: A classification for landscape mapping and conservation planning. The Southwestern Naturalist 45:385-420.

Hoagland, B.W. 2008. Vegetation of Oklahoma. In: Earth Sciences and Mineral Resources of Oklahoma. Johnson, K.S. and K.V. Luza (eds.). Norman (OK): Oklahoma Geological Survey.

Integrated Taxonomic Information System. 2015. http://www.itis.gov (12 February 2015).

Lindsey, R. 2008. Devastating drought settles on the High Plains. http://earthobservatory.nasa.gov (17 March 2015).

McPherson, J.K. 2003a. Black Mesa flora study. Oklahoma Native Plant Record 3:4-7.

McPherson, J.K. 2003b. Black Mesa flora study: Year two supplement. Oklahoma Native Plant Record 3:8-18.

National Drought Mitigation Center. 2015. http://droughtmonitor.unl.edu (17 March 2015).

National Oceanic and Atmospheric Administration, U.S. Department of Agriculture, and National Drought Mitigation Center. 2015. U.S. Drought Monitor. http://droughtmonitor.unl.edu/Home.aspx (9 March 2015).

NatureServe. 2015. NatureServe Explorer. http://www.natureserve.org/explorer (12 February 2015).

NOAA Center for Weather and Climate Prediction. 2015. http://www.cpc.noaa.gov (23 March 2015).

Oklahoma Climatological Survey. 2015. The Climate of Cimarron County. http://www.ocs.ou.edu (11 March 2015).

Oklahoma Natural Heritage Inventory. 2013. Plant Tracking List. http://www.oknaturalheritage.ou.edu (15 January 2013).
Oklahoma Natural Heritage Inventory. 2015. Heritage Database. http://www.oknaturalheritage.ou.edu (17 March 2015).

Oklahoma Vascular Plants Database. 2015. http://www.oklahomaplantdatabase.org (4 November 2015).

Rogers, C.M. 1953. The vegetation of the Mesa de Maya Region of Colorado, New Mexico and Oklahoma. Lloydia 16:257-290.

Rogers, C.M. 1954. Some botanical studies in the Black Mesa region of Oklahoma. Rhodora 56: 205-212.

South Central Climate Science Center. 2013. Drought history for the Oklahoma panhandle. http://www.southcentralclimate.org (17 March 2015).

Taylor, R.J. and C.E.S. Taylor. 1991. An Annotated List of the Ferns, Fern Allies, Gymnosperms and Flowering Plants of Oklahoma. Self-published.

Trewartha, G.T. 1968. An Introduction to Climate. New York: McGraw-Hill.

Tyrl, R.J., S.C. Barber, P. Buck, W.J. Elisens, J.R. Estes, P. Folley, L.K. Magrath, C.L. Murray, A.K. Ryburn, B.A. Smith, C.E.S. Taylor, R.A. Thompson, J.B. Walker, and L.E. Watson. 2010. Keys and Descriptions for the Vascular Plants of Oklahoma. Noble (OK): Flora Oklahoma Incorporated.

United States Department of the Interior, Fish and Wildlife Service. 1993. Federal Register, Part IV 58(188):51160.

United States Geological Survey. 2014. Summary of Cimarron County. http://regclim.coas.oregonstate.edu/N EXDCP30 app/data/counties 201403 31/summaries/40025/40025.pdf (23 March 2015).

USDA, NRCS. 2015. The PLANTS Database. http://plants.usda.gov/plants (9 March 2015).

Waterfall, U.T. 1949. Some results of a summer's botanizing in Oklahoma. Rhodora 51:18-28. 
Waterfall, U.T. 1950a. Some additions to the Oklahoma flora. Rhodora 52:19-24, 3541.
Waterfall, U.T. 1950b. Some results of a third summer's botanizing in Oklahoma. Rhodora 52:165-175. 


\title{
APPENDIX A
}

\section{List of Plant Taxa in Cimarron County and Black Mesa, Oklahoma}

Taxa list with duration, vegetation type, and nativity. $A=$ annual, $B=$ biennial, $P=$ =perennial;

AFSA=Artemisia filifolia shrubland association, BCBGBD=Bouteloua curtipendula-Bouteloua gracilisBouteloua dactyloides herbaceous association, $\mathrm{BGHJ}=$ Bouteloua gracilis-Hilaria jamesii herbaceous association, DAOF=Disturbed area/old field vegetation, HWV=herbaceous wetland vegetation, JMWA=Juniperus monosperma woodland alliance. An asterisk ( $\left.{ }^{*}\right)$ indicates a taxon that is non-native to the United States. A dagger $(\dagger)$ indicates a tracked taxon. Taxonomy follows the Integrated Taxonomic Information System (2015). Duration and nativity to Oklahoma were determined using the PLANTS Database (USDA-NRSC 2015); if the information from PLANTS was ambiguous, Taylor and Taylor (1991) was consulted. Vegetation classifications were based on Hoagland (2000).

\author{
Alismataceae \\ Alisma subcordatum Raf., P, HWV \\ Amaranthaceae \\ Amaranthus palmeri S. Watson, A, AFSA \\ Amaranthus tuberculatus (Moq.) J.D. Sauer, A, AFSA \\ Atriplex canescens (Pursh) Nutt., P, BGHJ \\ ${ }^{*}$ Chenopodium album L., A, BGHJ \\ Chenopodium berlandieri Moq., A, DAOF \\ Chenopodium incanum (S. Watson) A. Heller, A, BGHJ \\ Chenopodium leptophyllum (Moq.) Nutt. ex S. Watson, A, DAOF \\ Chenopodium pratericola Rydb., A, BGHJ \\ Chenopodium simplex (Torr.) Raf., A, BCBGBD \\ Chenopodium standleyanum Aellen, A, JMWA \\ Froelichia floridana (Nutt.) Moq., A, JMWA \\ *Kochia scoparia ssp. scoparia (L.) Schrad., A, DAOF \\ †Krascheninnikovia lanata (Pursh) A. Meeuse \& A. Smit, P, JMWA \\ Monolepis nuttalliana (Schult.) Green, A, DAOF \\ *Salsola tragus L., A, BCBGBD \\ Tidestromia lanuginosa (Nutt.) Standl., A, AFSA

\section{Amaryllidaceae} \\ Allium drummondii Regel, P, BGHJ

\section{Anacardiaceae} \\ Rhus aromatica Aiton var. pilosissima (Engl.) Shinners, $P, B G H J$ \\ Toxicodendron rydbergii (Small ex Rydb.) Greene, P, JMWA

\section{Apiaceae} \\ Cymopterus montanus Nutt. ex Torr. \& A. Gray, P, JMWA
}




\section{Apocynaceae}

Apocynum androsaemifolium L., P, JMWA

Asclepias asperula (Decne.) Woodson ssp. capricornu (Woodson) Woodson, P, JMWA

Asclepias engelmanniana Woodson, $\mathrm{P}, \mathrm{AFSA}$

Asclepias latifolia (Torr.) Raf., P, AFSA

†Asclepias macrotis Torr., $\mathrm{P}, \mathrm{JMWA}$

Asclepias subverticillata (A. Gray) Vail, P, AFSA

Asclepias viridiflora Raf., $\mathrm{P}, \mathrm{BCBGBD}$

\section{Asparagaceae}

${ }^{*}$ Asparagus officinalis L., P, BCBGBD

Yucca glauca Nutt., P, AFSA

\section{Asteraceae}

†Ambrosia confertiflora DC., P, AFSA

Ambrosia grayi (A. Nelson) Shinners, P, DAOF

Ambrosia psilostachya DC., P, DAOF

Ambrosia trifida L., A, BGHJ

Amphiachyris dracunculoides (DC.) Nutt., A, AFSA

†Artemisia carruthii Alph. Wood ex Carruth., P, BCBGBD

Artemisia filifolia Torr., P, AFSA

Artemisia ludoviciana Nutt., $\mathrm{P}, \mathrm{BGHJ}$

Baccharis salicina Torr. \& A. Gray, P, HWV

Berlandiera lyrata Benth., $P, A F S A$

†Brickellia brachyphylla (A. Gray) A. Gray, P, BGHJ

†Brickellia californica (Torr. \& A. Gray) A. Gray, P, BGHJ

†Brickellia eupatorioides (L.) Shinners var. chlorolepis (Woot. \& Standl.) B.L. Turner, P, BGHJ

Cirsium ochrocentrum A. Gray ssp. ochrocentrum, $\mathrm{P}, \mathrm{BGHJ}$

Cirsium undulatum (Nutt.) Spreng., P, BGHJ

Conyza canadensis (L.) Cronquist, A, DAOF

Diaperia prolifera (Nutt. ex DC.) Nutt., $A, B G H J$

Dyssodia papposa (Vent.) Hitchc., A, JMWA

Engelmannia peristenia (Raf.) Goodman \& C.A. Lawson, P, BGHJ

†Ericameria nauseosa (Pall. ex Pursh) G.L. Nesom \& Baird var. graveolens (Nutt.) Reveal \& Schuyler, $P$, JMWA

Erigeron bellidiastrum Nutt., AFSA, A

Erigeron flagellaris A. Gray, B, AFSA

Gaillardia pinnatifida Torr., P, BGHJ

Gaillardia pulchella Foug., A, BGHJ

Grindelia squarrosa (Pursh) Dunal, P, BGHJ

Gutierrezia sarothrae (Pursh) Britton \& Rusby, P, BGHJ

Helianthus annuus L., A, BGHJ

Helianthus ciliaris DC., $P, B C B G B D$

Helianthus petiolaris Nutt., A, DAOF

Heterotheca stenophylla (Gray) Shinners var. angustifolia (Rydb.) Semple, P, JMWA

Heterotheca subaxillaris (Lam.) Britton \& Rusby spp. latifolia (Buckley) Semple, A, BGHJ 
Heterotheca villosa (Pursh) Shinners var. villosa, P, JMWA

Hymenopappus flavescens A. Gray, B, AFSA

Hymenopappus tenuifolius Pursh, $\mathrm{B}, \mathrm{BGHJ}$

*Lactuca serriola L., A, DAOF

Liatris punctata Hook. var. punctata, $\mathrm{P}, \mathrm{AFSA}$

Lygodesmia juncea (Pursh) D. Don ex Hook., P, JMWA

Machaeranthera tanacetifolia (Kunth) Nees, A, JMWA

Melampodium leucanthum Torr. \& A. Gray, P, BGHJ

Packera plattensis (Nutt.) W.A. Weber \& A. Löve, P, BGHJ

Palafoxia sphacelata (Nutt. ex Torr.) Cory, A, BCBGBD

†Picradeniopsis woodhousei (A. Gray) Rydb., P, BGHJ

Pseudognaphalium canescens (DC.) W.A. Weber ssp. canescens, B, BGHJ

Ratibida columnifera (Nutt.) Woot. \& Standl., P, BCBGBD

Ratibida tagetes (James) Barnhart, $\mathrm{P}, \mathrm{DAOF}$

*Scorzonera laciniata L., P, DAOF

Senecio flaccidus Less. var. flaccidus, $\mathrm{P}, \mathrm{BGHJ}$

Senecio riddellii Torr. \& A. Gray, $P$, JMWA

Solidago gigantea Aiton, P, DAOF

†Solidago velutina DC. ssp. sparsiflora (A. Gray) Semple, P, BGHJ

Symphyotrichum subulatum (Michx.) G.L. Nesom, A, HWV

*Taraxacum officinale F.H. Wigg., P, DAOF

Tetraneuris acaulis (Pursh) Greene var. acaulis, $\mathrm{P}, \mathrm{JMWA}$

Tetraneuris scaposa (DC.) Greene var. scaposa, P, BGHJ

Thelesperma ambiguum A. Gray, P, AFSA

Thelesperma filifolium (Hook.) A. Gray, P, BGHJ

Thelesperma megapotamicum (Spreng.) Kuntze, P, BGHJ

Townsendia exscapa (Richardson) Porter, P, BGHJ

*Tragopogon dubius Scop., A, JMWA

Vernonia marginata (Torr.) Raf., P, JMWA

Xanthisma spinulosum (Pursh) D.R. Morgan \& R.L. Hartm. var. spinulosum, P, BGHJ

Xanthium strumarium L., A, HWV

Zinnia grandiflora Nutt., $\mathrm{P}, \mathrm{BGHJ}$

\section{Boraginaceae}

†Cryptantha cinerea (Greene) Cronquist var. jamesii (Torr.) Cronquist, P, AFSA,

Cryptantha minima Rydb., A, AFSA

Cryptantha thyrsiflora (Greene) Payson, P, BGHJ

Lappula occidentalis (S. Watson) Greene var. cupulata (A. Gray) Higgins, A, DAOF

Lappula occidentalis (S. Watson) Greene var. occidentalis, A, DAOF

Lithospermum incisum Lehm., P, BGHJ

Onosmodium bejariense DC. ex A. DC. var. occidentale (Mack.) B.L. Turner, P, JMWA

\section{Brassicaceae}

*Camelina microcarpa DC., A, BCBGBD

Descurainia pinnata (Walter) Britton ssp. brachycarpa (Richardson) Detling, A, JMWA

${ }^{*}$ Descurainia sophia (L.) Webb ex Prantl, A, DAOF

Erysimum asperum (Nutt.) DC., P, BGHJ 
Erysimum capitatum (Douglas ex Hook.) Greene, P, BGHJ

*Erysimum repandum L., A, BGHJ

*Lepidium densiflorum Schrad., A, DAOF

Physaria ovalifolia (Rydb.) O'Kane \& Al-Shehbaz ssp. ovalifolia, P, JMWA

Rorippa sinuata (Nutt.) Hitchc., P, HWV

*Sisymbrium altissimum L., A, BGHJ

\section{Cactaceae}

†Cylindropuntia imbricata (Haw.) F.M. Knuth, P, BGHJ

†Echinocereus reichenbachii (Terscheck ex Walp.) J.N. Haage, P, AFSA

$\dagger$ Echinocereus viridiflorus Engelm., P, JMWA

†Escobaria vivipara (Nutt.) Buxb., P, JMWA

Opuntia humifusa (Raf.) Raf. var. humifusa, P, BGHJ

Opuntia macrorhiza Engelm., P, JMWA

Opuntia phaeacantha Engelm., P, BGHJ,

tOpuntia polyacantha Haw.var. polyacantha, P, JMWA

\section{Cannabaceae}

Celtis reticulata Torr., P, BGHJ

\section{Caryophyllaceae}

Paronychia jamesii Torr. \& A. Gray, P, BGHJ

Paronychia sessiliflora Nutt., $\mathrm{P}, \mathrm{BGHJ}$

\section{Cleomaceae}

Polanisia dodecandra (L.) DC., A, BGHJ

\section{Commelinaceae}

Commelina erecta L., P, JMWA

Tradescantia occidentalis (Britton) Smyth var. occidentalis, P, BGHJ

\section{Convolvulaceae}

${ }^{*}$ Convolvulus arvensis L., BGHJ, $\mathrm{P}$

Convolvulus equitans Benth., BGHJ, $P$

†Cuscuta umbellata Kunth, A, DAOF

Evolvulus nuttallianus Schult., $\mathrm{P}, \mathrm{BGHJ}$

Ipomoea leptophylla Torr., P, BGHJ

\section{Crossomataceae}

†Glossopetalon spinescens A. Gray var. planitierum (Ensign) Yatsk., P, JMWA,

\section{Cucurbitaceae}

Cucurbita foetidissima Kunth, P, BGHJ

Cyclanthera dissecta (Torr. \& A. Gray) Arn., A, JMWA

\section{Cupressaceae}

†Juniperus monosperma (Engelm.) Sarg., P, JMWA 


\title{
Cyperaceae
}

Carex gravida L.H. Bailey, P, HWV

Carex muehlenbergii Schkuhr ex Willd., P, HWV

Schoenoplectus acutus (Muhl. ex Bigelow) Á. Löve \& D. Löve var. acutus, P, HWV

Schoenoplectus pungens (Vahl) Palla var. pungens, P, HWV

\author{
Euphorbiaceae \\ Croton texensis (Klotzsch) Müll. Arg., A, BGHJ \\ Ditaxis mercurialina (Nutt.) J.M. Coult., P, JMWA \\ Euphorbia dentata Michx., A, AFSA \\ Euphorbia exstipulata Engelm., A, BGHJ \\ Euphorbia fendleri Torr. \& A. Gray, P, JMWA \\ Euphorbia glyptosperma Engelm., A, AFSA \\ Euphorbia lata Engelm., $P, B G H$ \\ Euphorbia marginata Pursh, $A, B C B G B D$ \\ Euphorbia missurica Raf., A, BCBGBD \\ Euphorbia serpyllifolia Pers. var. serpyllifolia, A, BCBGBD \\ Tragia ramosa Torr., $\mathrm{P}$, JMWA
}

\section{Fabaceae}

Amorpha canescens Pursh, $\mathrm{P}, \mathrm{JMWA}$

Astragalus missouriensis Nutt., $\mathrm{P}, \mathrm{BGHJ}$

Astragalus mollissimus Torr., $\mathrm{P}, \mathrm{BGHJ}$

Dalea aurea Nutt. ex Fraser, $\mathrm{P}, \mathrm{BGHJ}$

Dalea candida Michx. ex. Willd var. oligophylla (Torr.) Shinners, P, JMWA

Dalea enneandra Nutt. ex Fraser, P, AFSA

†Dalea formosa Torr., $P$, JMWA

†Dalea jamesii (Torr.) Torr. \& A. Gray, P, BGHJ

Dalea lanata Spreng., $P, B G H J$

Dalea tenuifolia (A. Gray) Shinners, $P, B G H J$

Dalea villosa (Nutt.) Spreng., P, DAOF

†Desmanthus cooleyi (Eaton) Trel., P, BGHJ

Glycyrrhiza lepidota Pursh, P, BGHJ

†Hoffmannseggia drepanocarpa A. Gray, P, BGHJ

Hoffmannseggia glauca (Ortega) Eifert, P, BCBGBD

†Lupinus plattensis $\mathrm{S}$. Watson, $\mathrm{P}, \mathrm{AFSA}$

*Medicago sativa L., $\mathrm{P}, \mathrm{BGHJ}$

*Melilotus officinalis (L.) Lam., A, DAOF

Mimosa borealis A. Gray, P, BGHJ

Oxytropis lambertii Pursh, $P$, AFSA

Pediomelum cuspidatum (Pursh) Rydb., P, BGHJ

Prosopis glandulosa Torr. var. glandulosa, $\mathrm{P}, \mathrm{BGHJ}$

Psoralidium tenuiflorum (Pursh) Rydb., P, BGHJ

Robinia pseudoacacia L., P, DAOF

Sophora nuttalliana B.L. Turner, P, BGHJ 


\section{Fagaceae}

Quercus mohriana Buckley ex Rydb., P, JMWA

\section{Geraniaceae}

*Erodium cicutarium (L.) L'Hér. ex Aiton, A, DAOF

\section{Grossulariaceae}

Ribes aureum Pursh var. villosum DC., P, BCBGBD

†Ribes cereum Douglas, P, JMWA

\section{Juncaceae}

Juncus interior Wiegand, P, HWV

Juncus torreyi Coville, P, HWV

\section{Krameriaceae}

Krameria lanceolata Torr., P, BGHJ

\section{Lamiaceae}

Hedeoma drummondii Benth., P, BGHJ

*Marrubium vulgare L., $P, B G H J$

Monarda pectinata Nutt., A, AFSA

Salvia reflexa Hornem., A, JMWA

Teucrium laciniatum Torr., $P$, JMWA

\section{Linaceae}

Linum pratense (Norton) Small, $A, B G H J$

Linum rigidum Pursh var. rigidum, $A, B C B G B D$

\section{Loasaceae}

Mentzelia multiflora (Nutt.) A. Gray, A, AFSA

Mentzelia nuda (Pursh) Torr. \& A. Gray, P, AFSA

Mentzelia oligosperma Nutt. ex Sims, P, BGHJ

\section{Malvaceae}

Callirhoe involucrata (Torr. \& A. Gray) A. Gray, P, BCBGBD

*Malva neglecta Wallr., A, DAOF

†Sphaeralcea angustifolia (Cav.) G. Don, P, AFSA

Sphaeralcea coccinea (Nutt.) Rydb., P, BCBGBD

\section{Martyniaceae}

Proboscidea louisianica (Mill.) Thell., ssp. louisianica, A, AFSA

\section{Moraceae}

*Morus alba L., P, JMWA

\section{Nyctaginaceae}

†Abronia fragrans Nutt. ex Hook., P, AFSA 
Mirabilis albida (Walter) Heimerl, P, JMWA

Mirabilis linearis (Pursh) Heimerl var. subhispida (Heimerl) Spellenb., P, JMWA

Mirabilis nyctaginea (Michx.) MacMill., P, JMWA

\section{Oleaceae}

Forestiera pubescens Nutt., P, BGHJ

\section{Onagraceae}

Oenothera cespitosa Nutt., P, JMWA

Oenothera cinerea (Wooton \& Standl.) W.L. Wagner \& Hoch ssp. cinerea, P, BCBGBD

Oenothera curtiflora W.L. Wagner \& Hoch, A, DAOF

Oenothera hartwegii Benth. ssp. pubescens (A. Gray) W.L. Wagner \& Hoch, P, BGHJ

Oenothera serrulata Nutt., P, BCBGBD

Oenothera suffrutescens (Ser.) W.L. Wagner \& Hoch, P, BGHJ

Oenothera triloba Nutt., P, BGHJ

\section{Orobanchaceae}

Orobanche ludoviciana Nutt. ssp. multiflora (Nutt.) T.S. Collins ex H.L. White \& W.C. Holmes, A, BGHJ

\section{Papaveraceae}

$\dagger$ Argemone squarrosa Greene, P, BGHJ

Corydalis aurea Willd. ssp. occidentalis (Engelm. ex A. Gray) G.B. Ownbey, A, BCBGBD

\section{Pinaceae}

†Pinus edulis Engelm., P, JMWA

\section{Plantaginaceae}

Penstemon albidus Nutt., P, BGHJ

Penstemon ambiguus Torr., $\mathrm{P}, \mathrm{BGHJ}$

†Penstemon fendleri Torr. \& A. Gray, P, AFSA

Plantago patagonica Jacq., A, BGHJ

Veronica anagallis-aquatica L., P, HWV

\section{Poaceae}

${ }^{*}$ Aegilops cylindrica Host, A, DAOF

Andropogon gerardii Vitman ssp. hallii (Hack.) Wipff, P, AFSA

Andropogon gerardii Vitman ssp. gerardii, $\mathrm{P}, \mathrm{BCBGBD}$

Aristida adscensionis L., A, BGHJ

$\dagger$ Aristida arizonica Vasey, $\mathrm{P}, \mathrm{BGHJ}$

Aristida havardii Vasey, P, BCBGBD

Aristida oligantha Michx., A, AFSA

Aristida purpurascens Poir., P, BCBGBD

Aristida purpurea Nutt. var. purpurea, $\mathrm{P}, \mathrm{BGHJ}$

Bothriochloa barbinodis (Lag.) Herter, P, BGHJ

${ }^{*}$ Bothriochloa ischaemum (L.) Keng, $P$, AFSA

Bothriochloa laguroides (DC.) Herter, $\mathrm{P}, \mathrm{BGHJ}$

†Bouteloua barbata Lag., A, JMWA 
Bouteloua curtipendula (Michx.) Torr., P, AFSA

Bouteloua dactyloides (Nutt.) Columbus, P, BGHJ

†Bouteloua eriopoda (Torr.) Torr., P, BGHJ

Bouteloua gracilis (Kunth) Lag. ex Griffiths, P, BCBGBD

Bouteloua hirsuta Lag. , P, BGHJ

*Bromus arvensis L., A, DAOF

*Bromus catharticus Vahl, A, DAOF

*Bromus racemosus L., A, BGHJ

*Bromus tectorum L., A, DAOF

Calamovilfa gigantea (Nutt.) Scribn. \& Merr., P, BCBGBD

Cenchrus spinifex Cav., P, BGHJ

Chloris verticillata Nutt., $P, A F S A$

Chloris virgata Sw., A, BGHJ

${ }^{*}$ Cynodon dactylon (L.) Pers., P, DAOF

Distichlis spicata (L.) Greene var. stricta (Torr.) Thorne, P, BGHJ

Echinochloa muricata (P. Beauv.) Fernald, A, DAOF

Elymus canadensis L., P, BGHJ

Elymus elymoides (Raf.) Swezey, P, JMWA

Elymus virginicus L., P, AFSA

${ }^{\star}$ Eragrostis cilianensis (Bellardi) Vignolo ex Janch., A, AFSA

Erioneuron pilosum (Buckley) Nash, P, JMWA

†Hesperostipa neomexicana (Thurb.) Barkworth, P, BGHJ

†Hilaria jamesii (Torr.) Benth., P, BGHJ

Hopia obtusa (Kunth) Zuloaga \& Morrone, P, AFSA

Hordeum jubatum L., P, DAOF

Hordeum pusillum Nutt., A, DAOF

Leptochloa fusca (L.) Kunth spp. fasicularis N.W. Snow, A, HWV

Muhlenbergia asperifolia (Nees \& Meyen ex Trin.) Parodi, P, AFSA

Muhlenbergia paniculata (Nutt.) Columbus, P, DAOF

†Muhlenbergia phleoides (Kunth) Columbus, $\mathrm{P}, \mathrm{BGHJ}$

†Muhlenbergia porteri Scribn. ex Beal, P, JMWA

†Muhlenbergia torreyi (Kunth) Hitchc. ex Bush, P, BCBGBD

Munroa squarrosa (Nutt.) Torr., A, BGHJ

Panicum capillare L., A, DAOF

Panicum hallii Vasey, $\mathrm{P}, \mathrm{BGHJ}$

Panicum virgatum L., P, JMWA

Pascopyrum smithii (Rydb.) Barkworth \& D.R. Dewey, P, AFSA

Paspalum setaceum Michx. var. stramineum (Nash) D.J. Banks, P, DAOF

†Piptatherum micranthum (Trin. \& Rupr.) Barkworth, P, JMWA

Poa fendleriana (Steud.) Vasey, P, JMWA

*Polypogon monspeliensis (L.) Desf., A, HWV

Schizachyrium scoparium (Michx.) Nash, P, AFSA

Setaria macrostachya Kunth, $P$, DAOF

*Setaria viridis (L.) P. Beauv., A, DAOF

Sorghastrum nutans (L.) Nash, P, BGHJ

*Sorghum halepense (L.) Pers., P, BGHJ

Sporobolus airoides (Torr.) Torr. , P, BGHJ 
Sporobolus cryptandrus (Torr.) A. Gray, P, AFSA

Sporobolus pyramidatus (Lam.) Hitchc., P, AFSA

\section{Polemoniaceae}

Ipomopsis laxiflora (J.M. Coult.) V.E. Grant, A, JMWA

Polygalaceae

Polygala alba Nutt., P, BGHJ

Polygonaceae

Eriogonum annuum Nutt., A, AFSA

†Eriogonum jamesii Benth., P, BCBGBD

†Eriogonum lachnogynum Torr. ex Benth., P, BGHJ

†Eriogonum tenellum Torr., P, JMWA

Persicaria amphibia (L.) Delarbre, P, HWV

Persicaria lapathifolia (L.) Gray, A, HWV

*Polygonum aviculare L., A, DAOF

Rumex altissimus Alph. Wood, P, HWV

${ }^{*}$ Rumex crispus L., P, HWV

Rumex venosus Pursh, $\mathrm{P}, \mathrm{DAOF}$

\section{Portulacaceae}

Phemeranthus parviflorus (Nutt.) Kiger, P, AFSA

Portulaca oleracea L., A, JWMA

Portulaca pilosa L., A, DAOF

\section{Potamogetonaceae}

Zannichellia palustris L., P, HWV

\section{Pteridaceae}

Cheilanthes eatonii Baker, P, JMWA

Notholaena standleyi, P, JMWA

\section{Ranunculaceae}

Delphinium carolinianum Walter ssp. virescens (Nutt.) R.E. Brooks, P, JMWA

Ranunculus abortivus L., P, HWV

Ranunculus sceleratus L., A, HWV

*Ranunculus testiculatus Crantz, A, DAOF

\section{Rosaceae}

†Cercocarpus montanus Raf., P, JMWA

Prunus virginiana $L$. var. demissa (Nutt.) Torr., P, JMWA

†Rubus deliciosus Torr., P, JMWA

\section{Rutaceae}

Ptelea trifoliata L., P, JMWA 
Volume 15, December 2015

\section{Salicaceae}

Populus deltoides W. Bartram ex Marshall, P, HWV

Salix amygdaloides Andersson, $\mathrm{P}, \mathrm{HWV}$

Salix exigua Nutt., P, HWV

Salix nigra Marshall, $\mathrm{P}, \mathrm{HWV}$

\section{Santalaceae}

Comandra umbellata (L.) Nutt. ssp. pallida (A. DC.) Piehl, P, JMWA

\section{Sapindaceae}

Sapindus saponaria L. var. drummondii (Hook. \& Arn.) L.D. Benson, P, DAOF

\section{Selaginellaceae}

†Selaginella underwoodii Hieron., P, JMWA

\section{Solanaceae}

Chamaesaracha coniodes (Moric. ex Dunal) Britton, P, JMWA

Datura quercifolia Kunth, A, DAOF

Physalis hederifolia A. Gray var. fendleri (A. Gray) Cronquist, P, JMWA

Physalis longifolia Nutt. var. longifolia, P, AFSA

Quincula lobata (Torr.) Raf., P, JMWA

Solanum elaeagnifolium Cav., $P$, DAOF

Solanum ptychanthum Dunal, A, BCBGBD

Solanum rostratum Dunal, A, AFSA

Solanum triflorum Nutt., A, DAOF

\section{Tamaricaceae}

*Tamarix chinensis Lour., P, HWV

\section{Verbenaceae}

Glandularia bipinnatifida (Nutt.) Nutt. var. ciliata (Benth.) B.L. Turner, A, BGHJ

Glandularia canadensis (L.) Nutt., P, JMWA

Glandularia pumila (Rydb.) Umber, A, BGHJ

Phyla cuneifolia (Torr.) Greene, P, HWV

Verbena bracteata Cav. ex Lag. \& Rodr., A, AFSA

\section{Violaceae}

Hybanthus verticillatus (Ortega) Baill., P, BGHJ

\section{Vitaceae}

Vitis vulpina L., P, JMWA

\section{Zygophyllaceae}

Kallstroemia parviflora Norton, A, AFSA

${ }^{\star}$ Tribulus terrestris L., A, AFSA 


\section{APPENDIX B}

\section{List of Plant Taxa in Cimarron County and Black Mesa, Oklahoma Not Found by Buthod and Hoagland}

Taxa from the published lists of Rogers (1953), McPherson (2003a, b), and Folley (2003) that were not found by Buthod and Hoagland. R=Rogers collection, M=Mcpherson collection, F=Folley collection. Taxonomy has been updated and follows the Integrated Taxonomic Information System (2015).

\section{Amaranthaceae \\ Amaranthus retroflexus L., M Chenopodium albescens Small, $\mathrm{R}$ Cycloloma atriplicifolium (Spreng.) J.M. Coult., R}

Froelichia gracilis (Hook.) Moq., R Guilleminea densa (Humb. \& Bonpl. ex Schult.) Moq. var. densa, R Salsola kali L. ssp. tenuifolia Moq., M Suckleya suckleyana (Torr.) Rydb., M

\section{Amaryllidaceae}

Allium canadense $\mathrm{L}$. var. fraseri Ownbey, $\mathrm{M}$

\section{Anacardiaceae}

Rhus aromatica Aiton var. simplicifolia (Greene) Cronquist, $\mathrm{R}$

Toxicodendron radicans (L.) Kuntze, $\mathrm{M}$

\section{Apiaceae}

Cymopterus glomeratus (Nutt.) DC., M

\section{Apocynaceae}

Asclepias arenaria Torr. , $\mathrm{M}$

Asclepias involucrata Engelm. ex Torr. , $\mathrm{R}$

Asclepias pumila (A. Gray) Vail, R, M

Asclepias uncialis Greene, $\mathrm{M}$

Funastrum crispum (Benth.) Schltr., R, M

\section{Araceae}

Lemna minor L., M

\section{Asparagaceae}

Nolina texana S. Watson, F (collections are actually Nolina greenei S. Watson ex Trel.; Hess 2002))

Yucca harrimaniae Trel., $\mathrm{F}$

\section{Aspleniaceae}

Asplenium septentrionale (L.) Hoffm., M

\section{Asteraceae}

Antennaria parvifolia Nutt., $\mathrm{R}$ 
Artemisia dracunculus L., $\mathrm{R}, \mathrm{M}$

Baccharis wrightii A. Gray, $\mathrm{R}$

Bidens cernua L., $\mathrm{F}$

Brickellia eupatorioides (L.) Shinners var. corymbulosa (Torr. \& A. Gray) Shinners, R

Chaetopappa ericoides (Torr.) G.L. Nesom, R, M

Ericameria nauseosa (Pall. ex Pursh) G.L. Nesom \& Baird var. nauseosa, R, M

Erigeron nudiflorus Buckley, R

Erigeron tracyi Greene, M

Nothocalais cuspidata (Pursh) Greene, M

Oonopsis foliosa (A. Gray) Greene var. foliosa, R

Packera tridenticulata (Rydb.) W.A. Weber \& A. Löve, R, M

Pericome caudata A. Gray, R, M, F

Picradeniopsis oppositifolia (Nutt.) Rydb. ex Britton, R

Psilostrophe villosa Rydb., $\mathrm{F}$

Solidago mollis Bartlett , M

Solidago petiolaris Aiton, $\mathrm{M}$

Stephanomeria pauciflora (Torr.) A. Nelson, R, M

Symphyotrichum ericoides (L.) G.L. Nesom, R, M

Symphyotrichum fendleri (A. Gray) G.L. Nesom, M

Symphyotrichum oblongifolium (Nutt.) G.L. Nesom, M

Verbesina encelioides (Cav.) Benth. \& Hook. f. ex A. Gray, M

Vernonia fasciculata Michx., F

Xanthisma spinulosum (Pursh) D.R. Morgon \& R.L. Hartm. var. glaberrimum (Rydberg) D.R. Morgan \&

R.L. Hartm., R

\section{Boraginaceae}

Cryptantha cinerea (Greene) Cronquist var. cinerea, $R$

Cryptantha crassisepala (Torr. \& A. Gray) Greene, R

Euploca convolvulacea Nutt., $\mathrm{F}$

Lithospermum multiflorum Torr. ex A. Gray, F

\section{Brassicaceae}

Boechera fendleri (S. Watson) W.A. Weber, M

\section{Cactaceae}

Opuntia fragilis (Nutt.) Haw., $\mathrm{F}$

\section{Campanulaceae}

Lobelia cardinalis L., F

\section{Cleomaceae}

Peritoma serrulata (Pursh) DC., R, F

Polanisia jamesii (Torr. \& A. Gray) Iltis, F

\section{Cupressaceae}

Juniperus scopulorum Sarg., M 


\section{Cyperaceae}

Carex brevior (Dewey) Mack. , F

Cyperus croceus Vahl, $\mathrm{F}$

Cyperus schweinitzii Torr., R, M

Schoenoplectus tabernaemontani (C.C. Gmel.) Palla, M

Scirpus atrovirens Willd., $\mathrm{F}$

Scirpus pallidus (Britton) Fernald, $R$

\section{Cystopteridaceae}

Cystopteris fragilis (L.) Bernh., F

\section{Equisetaceae}

Equisetum laevigatum $\mathrm{A}$. Br., $\mathrm{R}$

\section{Euphorbiaceae}

Ditaxis humilis (Engelm. \& A. Gray) Pax, R, M

Euphorbia geyeri Engelm., $\mathrm{R}$

Euphorbia spathulata Lam., R

\section{Fabaceae}

Astragalus ceramicus E. Sheld., $\mathrm{F}$

Astragalus crassicarpus Nutt., $\mathrm{R}$

Astragalus crassicarpus Nutt. var. paysonii (E.H. Kelso) Barneby, M

Astragalus gracilis Nutt., $\mathrm{R}$

Astragalus hallii A. Gray, $\mathrm{R}$

Astragalus lotiflorus Hook. , R, M

Astragalus puniceus Osterh., M

Colutea arborescens L., F

Dalea candida Michx. ex Willd var. candida, $\mathrm{R}$

Dalea compacta Spreng. var. compacta, $\mathrm{R}$

Dalea nana Torr. ex A. Gray, R

Dalea purpurea Vent. var. purpurea, $\mathrm{R}$

Hedysarum boreale Nutt., $\mathrm{R}$

Melilotus albus Medik., $\mathrm{R}$

Pediomelum argophyllum (Pursh) J.W. Grimes, M

Pediomelum hypogaeum (Nutt.) Rydb. var. hypogaeum, R

Pomaria jamesii (Torr. \& A. Gray) Walp., R, M

Vicia americana Muhl. ex Willd. , M

Vicia ludoviciana Nutt. ex Torr. \& A. Gray var. leavenworthii (Nutt. ex Torr. \& A. Gray) Broich, R

\section{Fagaceae}

Quercus gambelii Nutt., R

Quercus grisea Liebm., R

Quercus X undulata Torr., $\mathrm{R}$

\section{Lamiaceae}

Salvia azurea Michx. ex Lam. var. grandiflora Benth., M 


\section{Linaceae}

Linum lewisii Pursh , R, M

Loasaceae

Mentzelia decapetala (Pursh ex Sims) Urb. \& Gilg, R, M

\section{Lythraceae}

Lythrum alatum Pursh, $\mathrm{R}$

\section{Nyctaginaceae}

Mirabilis glabra (S. Watson) Standl., R, M

Mirabilis linearis (Pursh) Heimerl var. linearis, $\mathrm{R}$

\section{Onagraceae}

Oenothera albicaulis Pursh, $\mathrm{R}$ Oenothera engelmannii (Small) Munz, R, F Oenothera lavandulifolia Torr. \& A. Gray, M Oenothera pallida Lindl. ssp. latifolia (Rydb.) Munz, F

\section{Orobanchaceae}

Castilleja sessiliflora Pursh, R, M

\section{Papaveraceae}

Argemone polyanthemos (Fedde) G.B. Ownbey, R

\section{Plantaginaceae}

Penstemon angustifolius Nutt. ex Pursh var. caudatus (A. Heller) Rydb., R

\section{Poaceae}

Achnatherum hymenoides (Roem. \& Schult.) Barkworth, R, M Achnatherum scribneri (Vasey) Barkworth, R, M

Andropogon virginicus L., $\mathrm{F}$

Aristida barbata E. Fourn., R

Aristida divaricata Humb. \& Bonpl. Ex Willd., R

Aristida purpurea Nutt. var. fendleriana (Steud.) Vasey, $\mathrm{R}$

Aristida purpurea Nutt. var. longiseta (Steud.) Vasey, R

Aristida purpurea Nutt. var. wrightii (Nash) Allred, R, M

Bothriochloa saccharoides (Sw.) Rydb., M

Bouteloua hirsuta Lag. var. hirsuta, M

Bromus japonicus Thunb. ex Murray, $R$

Bromus lanatipes (Shear) Rydb., R, M

Cenchrus incertus M.A. Curtis, $R$

Cenchrus longispinus (Hack.) Fernald, M

Dichanthelium oligosanthes (Schult.) Gould, R

Digitaria californica (Benth.) Henrard, R

Digitaria cognata (Schult.) Pilg., R

Echinochloa crus-galli (L.) P. Beauv., M 
Enneapogon desvauxii P. Beauv., R

Eragrostis curtipedicellata Buckley, $\mathrm{R}$

Eragrostis intermedia Hitchc., $\mathrm{R}$

Eragrostis secundiflora J. Presl, R

Eragrostis sessilispica Buckley, $\mathrm{R}$

Eragrostis trichodes (Nutt.) Alph. Wood, M

Hesperostipa comata (Trin. \& Rupr.) Barkworth, R, M

Leptochloa dubia (Kunth) Nees, R

Muhlenbergia arenicola Buckley, $\mathrm{R}$

Muhlenbergia racemosa (Michx.) Britton, Sterns \& Poggenb., R, F

Phalaris caroliniana Walter, $\mathrm{R}$

Phragmites australis (Cav.) Trin. ex Steud., $\mathrm{R}$

Poa nemoralis L., $\mathrm{R}$

Poa pratensis L., $\mathrm{R}$

Setaria leucopila (Scribn. \& Merr.) K. Schum., M

Sphenopholis obtusata (Michx.) Scribn., R

Tridens muticus (Torr.) Nash var. elongatus (Buckley) Shinners, $R$

Triplasis purpurea (Walter) Chapm., $\mathrm{R}$

Vulpia octoflora (Walter) Rydb., R, M

Polemoniaceae

Giliastrum rigidulum (Benth.) Rydb., $\mathrm{F}$

Polygonaceae

Polygonum ramosissimum Michx. , M

\section{Pteridaceae}

Astrolepis sinuata (Lag. ex Sw.) D.M. Benham \& Windham ssp. sinuata, R

Cheilanthes feei T. Moore, R, M

Cheilanthes lanosa (Michx.) D.C. Eaton, M

Pellaea atropurpurea (L.) Link, R, M

\section{Ranunculaceae}

Clematis hirsutissima Pursh var. scottii (Porter) R.O. Erickson, M

Ranunculus cymbalaria Pursh, $\mathrm{R}$

\section{Rhamnaceae}

Ceanothus herbaceus Raf., $\mathrm{R}$

\section{Rosaceae}

Fallugia paradoxa (D. Don) Endl. ex Torr., R

Physocarpus monogynus (Torr.) J.M. Coult., R, M

Prunus americana Marshall , $\mathrm{M}$

Rosa woodsii Lindl., $\mathrm{F}$

\section{Rubiaceae}

Galium texense A. Gray, M 
Volume 15, December 2015

\section{Salicaceae}

Salix interior Rowlee, M

Selaginellaceae

Selaginella densa Rydb., R

Solanaceae

Solanum nigrum L., $\mathrm{R}$

Tamaricaceae

Tamarix gallica L., R, M

Urticaceae

Parietaria pensylvanica Muhl. ex Willd., M

Verbenaceae

Verbena plicata Greene, R

Vitaceae

Parthenocissus quinquefolia (L.) Planch., M Vitis acerifolia Raf., R, F,

Woodsiaceae

Woodsia oregana D.C. Eaton, R, M 